Lymphology 53 (2020) 76-80

\title{
LYMPHATIC SYSTEM MALFORMATIONS IN NOONAN SYNDROME: TWO CASE REPORTS AND IMAGING ANALYSIS
}

\author{
N.-F. Liu, M.-Z. Gao
}

Department of Plastic \& Reconstructive Surgery, Shanghai Ninth People's Hospital, Shanghai Jiao Tong University School of Medicine, Shanghai, China

\section{ABSTRACT}

L ymphedema is a well-known complication of Noonan syndrome (NS) but the lymphatic malformations in NS are poorly understood. We report clinical, genetic, and imaging information about a boy and girl with NS and late-onset lower extremity lymphedema. A de novo mission mutation of RIT1 (NM_006912.5) c.246T >A, p.Phe82Leu was identified in the girl, who also showed systemic lymphatic hyperplasia and dysfunction. Magnetic resonance lymphangiography (MRL) of the boy clearly demonstrated segmental dilated and hyperplastic lymphatics with impaired transport function in an affected limb and pelvic region. Indocyanine green lymphography (ICGL) showed delayed and partial enhancement of the lymph vessels in the affected limb but no lymph reflux was detected. No causative mutation was identified in the second case. Lymphoscintigraphy (LSG) failed to show lymph vessels in either of the children. Our study showed that MRL is a reliable and accurate test that can be used to demonstrate morpho-logical and functional defects of the lymphatic system. Moreover, ICGL is sufficiently sensitive to determine the functional condition of peripheral lymph vessels. The combined use of imaging modalities can give an accurate diagnosis of complex lymphatic system anomalies in NS and other syndromic diseases.
Keywords: lymphedema, Noonan syndrome, magnetic resonance lymphangiography, lymphoscintigraphy, indocyanine green lymphography, lymphatic malformation, RIT1

Noonan syndrome (NS) is a relatively common disease with a prevalence of $1 / 1000-2500$ live births (1-3). It is an inherited multisystem disorder associated with mutations in genes encoding the Ras/MAPK pathway, which explain about $70 \%$ of cases $(4,5)$. Somatic mosaicism may be present in some if the remaining $30 \%$ of cases. Clinical manifestations include congenital heart disease such as pulmonary stenosis and hypertrophic cardiomyopathy, growth failure as short stature, mild learning difficulties, distinct facial features such as downward-slanting palpebral fissures, ptosis, low-set posteriorly rotated ears, webbed neck, and bleeding tendency. Lymphedema is reported in $20 \%-49 \%$ of NS patients $(6,7)$ in extremities, genital regions, or viscera at birth or later in childhood. However, the nature of lymphatic anomalies in NS has not been studied in detail. Here, we present clinical, genetic, and multiimaging investigations of two NS children with lower extremity lymphedema, including results from magnetic resonance lymphangiography (MRL), lymphoscintigraphy (LSG), and indocyanine green lymphography (ICGL). 


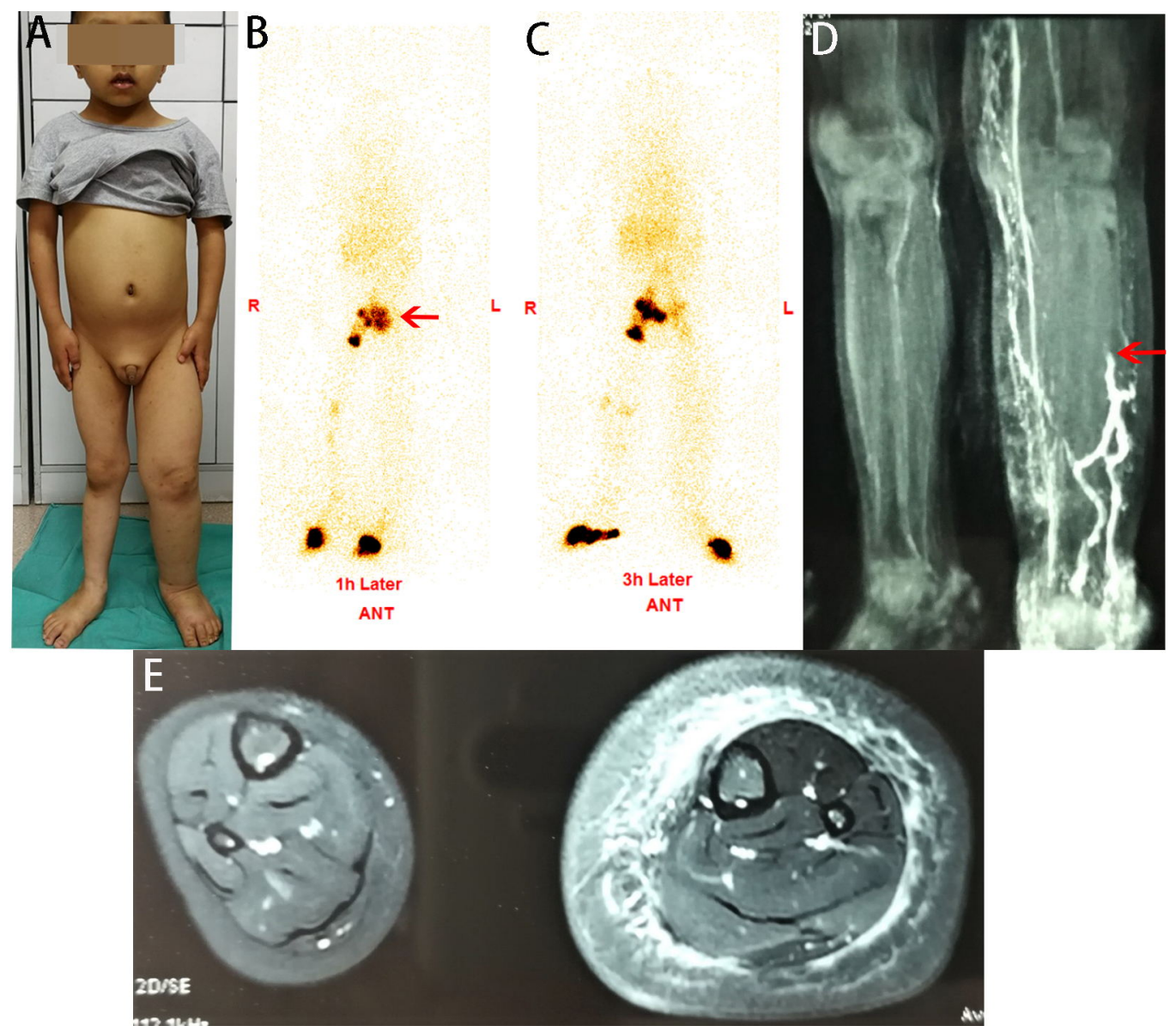

Fig. 1. A) A 5-year-old boy with NS with distinct facial features and lymphedema in the pubic region and left lower limb. B,C) LSG did not show lymphatics or inguinal lymph nodes in the affected limb after 1 (arrow) and $3 \mathrm{hrs.}$ D) MRL demonstrated dilated lymph collectors in the medial side of the left lower limb and greatly dilated lymph vessels that ascended only halfway up the calf (arrow). E) MRL showed massive edema in the subcutaneous layer of the left calf.

\section{CASE 1}

A 5-year-old boy was diagnosed with NS with pulmonary stenosis, atrial septal defects, and bilateral hypertrophic cardiomyopathy after birth with distinct facial features including widespaced eyes, a broad nose with a depressed root, low-set and posteriorly rotated ears, and hyperopia. He had surgery for pulmonary stenosis at the age of 2 . Lymphedema of his left leg occurred 18 months ago and progressed to his thigh and pubic region. At our clinic, thickening of the skin of his toes was obvious in the affected limb together with skin hyperpigmentation. Following contrast injection in the affected limb, no lymphatic channel was observed on the LSG images after 1 and 3 hours. Very weak tracer accumulation was seen in the inguinal region after 3 hours compared with strong tracer uptake in the inguinal popliteal and lymph nodes in the contralateral non-edematous limb. MRL clearly demonstrated 4-5 lymph collectors ascending to the groin on the medial side and two clearly dilated lymphatics ascending slowly then stopping halfway up the lower leg on the lateral side, suggesting impaired transportation of lymph vessels (Fig. 1). ICGL 

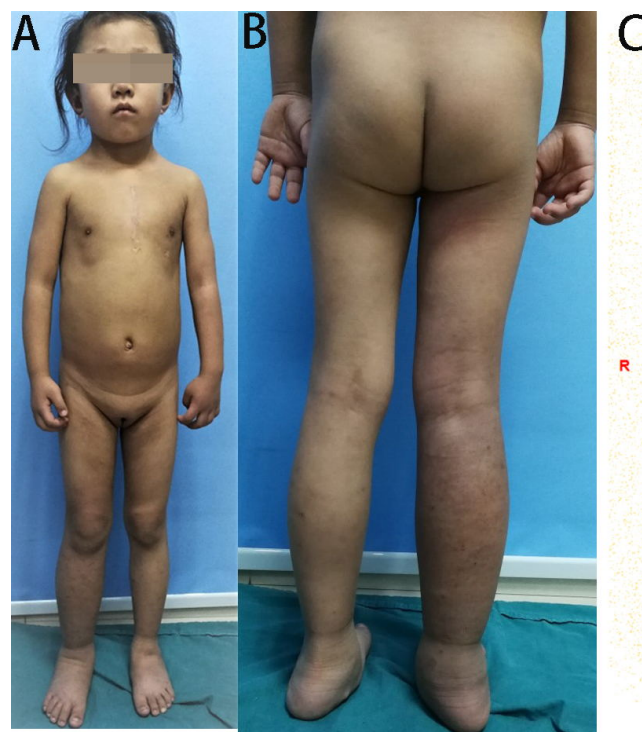

D
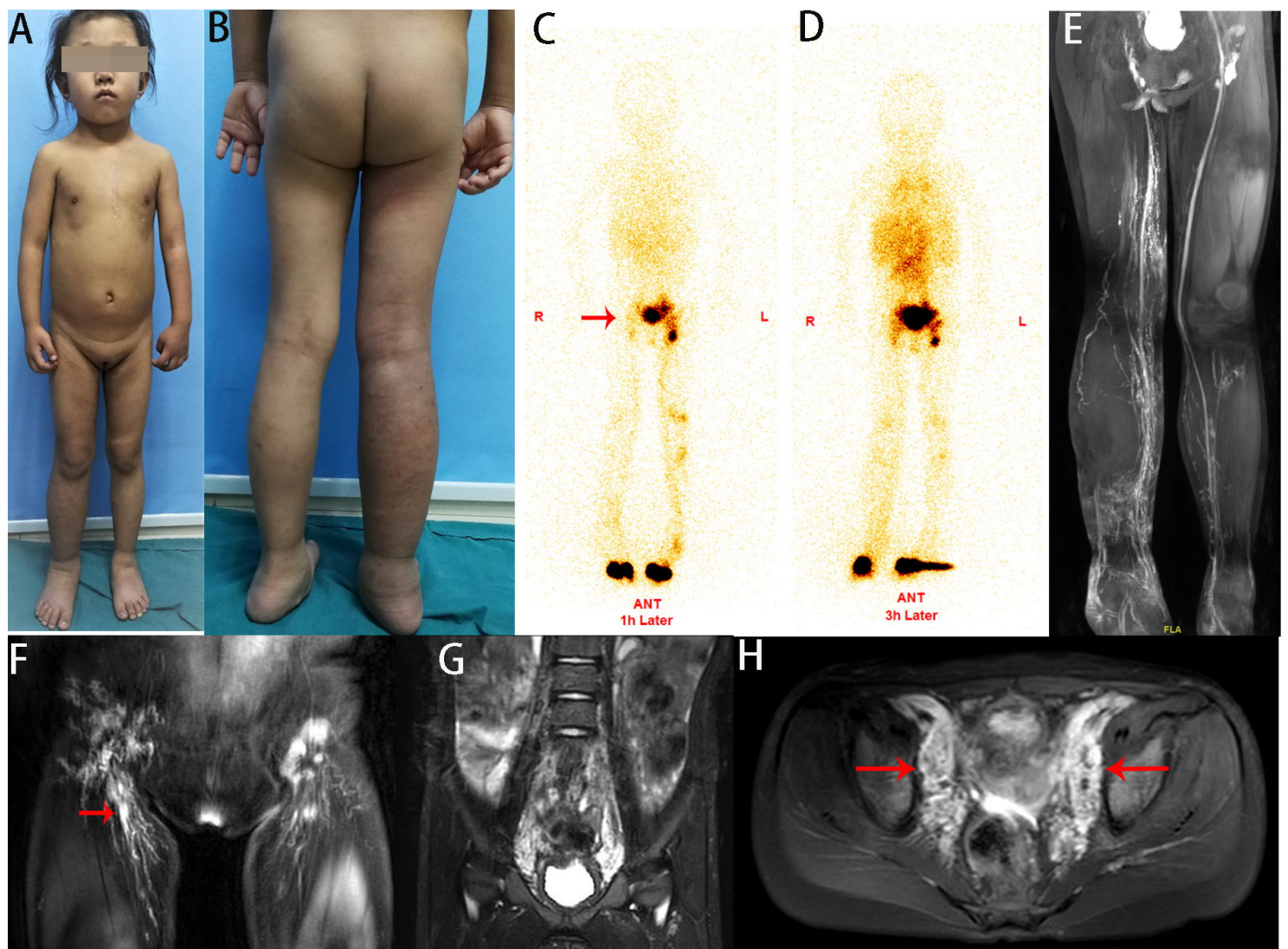

$\mathrm{H}$

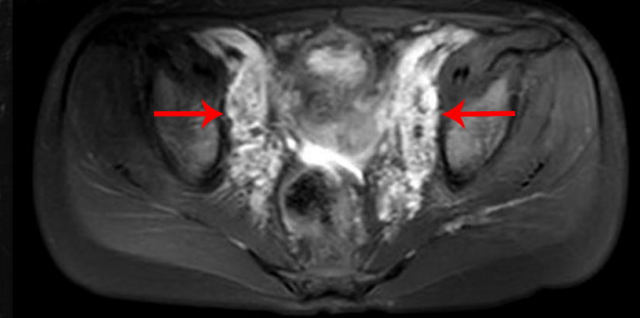

Fig. 2. A,B) A 5-year-old girl with characteristic facial deformation of NS and lymphedema of her right lower extremity, pubic, and left groin regions. C) LSG showed unclear lymph vessels and lymph nodes in the right affected lower limb after $1 \mathrm{hr}$ (arrow). D) Additional isotopic accumulated in the right inguinal and abdominal region after $3 \mathrm{hrs.}$ E) MRL demonstrated dilated lymph collectors in the entire length of the right lower limb. F) Prominent afferent and efferent lymphatics of bilateral inguinal lymph nodes, and only enhanced lower group lymph nodes (arrow) compared with full contrast enhancement in the lymph nodes of the left side. G,H) Hyperplasia of lymph vessels in the bilateral iliac and pelvic regions (arrows).

revealed delayed and partial enhancement of the lymph collector in the distal region of the calf. However, no lymph reflux was detected in the clearly dilated lymph vessels during ICGL inspection. No venous disease was detected by duplex scanning. No known pathogenic mutations were identified by whole exome sequencing of DNA extracted from the patient's blood sample.

\section{CASE 2}

A 5-year-old girl with a prior diagnosis of NS based on a congenital heart anomaly (pulmonary stenosis and bilateral hypertrophic cardiomyopathy), characteristic facial deforming, and irregular vaginal bleeding attended our clinic with non-pitting edema of her right leg for 5 months with skin hyperpigmentation and papillomatosis. Lymphedema was also exhibited in her pubic and groin regions. She had undergone surgery for pulmonary stenosis at the age of 2.5 years. No abnormal findings regarding blood cells, coagulation factors, thromboplastin time, platelet aggregation, or female hormones were identified. Genetic analysis identified a de novo missense mutation of RIT1 
(NM_006912.5) c.246T>A, p.Phe82Leu which was previously reported as a pathogenic mutation of NS in the ClinVar database (RCV000408903.2, RCV000427451.2). No venous anomaly was detected in her lower limbs by duplex scanning, but LSG revealed faint lymph vessels and inguinal lymph nodes in the affected right lower limb 1 hour after contrast injection. Additional isotopic tracer accumulated in the lymph nodes after 3 hours, but much less than in the contralateral limb. MRL clearly showed dilated lymph collectors in the right lower extremity, and prominent afferent and efferent lymphatics of the bilateral inguinal lymph nodes. Only the lymph nodes of the lower group of inguinal lymph nodes were enhanced, leaving middle and upper groups unfilled with contrast, suggesting impaired afferent lymphatic transportation (Fig. 2). MRL also demonstrated obvious hyperplasia of iliac lymph collectors and lumbar trunks.

\section{DISCUSSION}

Lymphedema is a well-known complication of NS that is attributed to lymphatic hypoplasia and dysplasia (8) but the lymphatic malformations have been poorly studied. Lymphatic system malformations in NS are congenital but lymphedema may occur after birth (1). Indeed, the onset of lower limb lymphedema in the current patients was at the age of 4 and 5 years, shortly after heart surgery. However, the progression of lymphedema was rapid, with short histories of 5 and 18 months, respectively; both patients exhibited stage III lymphedema with skin keratinization. This indicates that early diagnosis is important to control the development of disorder.

In the present study, we evaluated lymphatic anomalies in two NS patients with lower extremity lymphedema using multiimage methods and identified systemic lymphatic hyperplasia and dysfunction in a child with an RIT1 mutation, and segmental lymphatic dilatation and impaired transportation function in another child with no causative gene detected. We first used LSG but this failed to identify lymphatic collectors or inguinal lymph nodes in the affected limbs of either patient after 1 hour. Very faintly enhanced lymph nodes were visible after 3 hours, but no lymph vessel could be clearly seen. This could be for two reasons: first, the initial lymph vessel might have been unable to take up the intradermally-injected isotopic tracer, and second, lymphatic hypoplasia or aplasia may have occurred in the affected limb. We then used MRL which clearly showed dilated, tortuous and hyperplastic collecting lymph vessels in the affected limbs of both patients and in the inguinal and pelvic regions of the affected girl. MRL also revealed the impairment of lymphatic functions as the partial enhancement of large lymph collectors in the distal part of the affected lower limb. Additionally, the slow and partial filling of the contrast in inguinal lymph nodes evidenced only by lymph nodes of the lower group was enhanced in the affected limb, compared with full enhancement of the contrast in all superficial lymph nodes of the nonlymphedematous limb. MRL imaging suggested a hyperplastic and functional impairment of the lymphatics in both patients. Further study with ICGL showed the slow and partial demonstration of lymph vessels in the affected limbs, suggesting impaired lymph flow.

Comparing the three image modalities, MRL was found to be the most reliable and accurate in showing both morphological and functional defects of the lymphatics and lymph nodes (9). LSG examination had the disadvantage of failure to take up the injected tracer which may cause confusion in the diagnosis of abnormal lymphatics (10), while ICGL was sensitive in detecting the lymphatic function by its real-time observation of the lymph flow, which enables the detection of lymphatic pathology such as lymph reflux. The combination of the imaging tests can help to outline complicated lymphatic defects occurring in NS and other syndromic disease. 


\section{ACKNOWLEDGMENTS}

National Nature and Science Foundation of China (Grant number: 81272146)

\section{CONFLICT OF INTEREST AND DISCLOSURE}

The authors declare no competing financial interests exist.

\section{REFERENCES}

1. Noonan, JA: Hypertelorism with Turner phenotype. A new syndrome with associated congenital heart disease. Am. J. Dis. Child. 116 (1968), 373-380.

2. Joyce, S, K Gordon, G Brice, et al: The lymphatic phenotype in Noonan and Cardiofaciocutaneous syndrome. Eur. J. Hum. Genet. 24 (2016), 690-696.

3. Jones, GE, S Mansour: An approach to familial lymphedema. Clin. Med. 17 (2017), $552-557$

4. Romano, AA, JE Allanson, J Dahlgren, et al: Noonan syndrome: Clinical features, diagnosis and management guidelines. Pediatrics 126 (2010), 746-759.

5. Ding, Y, XY Hu, YN Song, et al: A report on a girl of Noonan syndrome 9 presenting with bilateral lower limbs lymphedema. Chin. Med. J. 132 (2019), 480-482.
6. Rauen, KA: The RASo pathies. Annu. Rev. Genomics Hum. Genet. 14 (2013), 355-369.

7. Smpokou, P, E Tworog-Dube, RS Kucherlapati, et al: Medical complications, clinical findings and educational outcomes in adults with Noonan syndrome. Am. J. Med. Genet. Part A 158A (2012), 3106-3111.

8. Hasegawa, K, Y Nagaoka, H Maruyama, et al: Late-onset lymphedema and proteinlosingeEnteropathy with Noonan syndrome. Clin. Pediatr. Endocrinol. 18 (2009), 87-93.

9. Liu, NF, Q Lu, ZH Jiang, et al: Anatomic and functional evaluation of the lymphatics and lymph nodes in diagnosis of lymphatic circulation disorders with contrast magnetic resonance lymphangiography. J. Vasc. Surg. 49 (2009), 980-987.

10. Liu, NF, Q Lu, PA Liu, et al: Comparison of radionuclide lymphoscintigraphy and dynamic magnetic resonance lymphangiography for investigating extremity lymphedema. Br. J. Surg. 97 (2010), 359-365.

\author{
Ningfei Liu MD PhD \\ Department of Plastic \& Reconstructive \\ Surgery \\ Shanghai Ninth People's Hospital \\ 639 Zhi Zao Ju Road \\ Shanghai, 200011, China \\ Tel: +86-21-23271699 x 5734 \\ Fax: +86-21-53078128 \\ E-mail: liuningfei@126.com
}

